\title{
Two types of portosystemic shunts of the dog - a clinical
}

case

\author{
Georgi Georgiev ${ }^{1}$, Monika Stefanova ${ }^{2}$, Vasil \\ Manov3.
}

1. Department of Anatomy, histology and physiology, Faculty of Veterinary Medicine, University of Forestry Sofia, Bulgaria;

2. Blue Sky Commerce, Sofia, Bulgaria;

3. Department of Obstetrics, pathologic anatomy and biochemistry, Faculty of Veterinary Medicine, University of Forestry - Sofia, Bulgaria;

\begin{abstract}
Recently reports of research on vascular abnormalities in domestic animals have increased in the literature. With the expansion of diagnostic imaging methods more various malformations of the portal vein in dogs of different breed affiliation can be detected.

In these cases, the liver is most often affected and clinical vascular anomalies are manifested with hepatic encephalopathy. They appear with nonspecific symptoms which are hard to differentiate from other disorders of the nervous and digestive system. The different types of portal shunts should be known and researched in dog's veterinary practice. It is possible to visualize them by contrasting methods of imaging diagnostics, and it can be estimated whether they are operable.
\end{abstract}

Keywords: dog, vessels anomaly, liver, portal vein, shunt

\section{Background}


Portosystemic shunts (PSS) are represented as an anomaly in development of venous blood vessels of the liver. The portal vein of mammals is formed from four large venous inlets $-v$. lienalis, $v$. gastroduodenalis, v. mesenterica cranialis and v. mesenterica caudalis $(5,11)$. The different types of portal shunts are known as derivations of this large inlets or their smaller branches towards caudal vena cava, in which bypasses of the liver portal system are located. PSS are intra- and extrahepatal, single and multiple, congenital and obtained. The different types of PSS in humans have been researched more deeply and thoroughly. The most frequent congenital PSS are six - patent venous duct, porto-caval shunt, porto-azygos, shunt, porto-azygos with interruption of the prerenal part of the inferior vena cava, hypoplasia of portal vein with developed multiple collateral communications and microvascular dysplasia $(1,3,4,6,10,14,15)$. The obtained PSS are less frequent and are due to the liver's cirrhotic amendments and hypoplasia of the portal vein. Multiple extrahepatal shunts from the remaining nonfunctional vessels of the portal venous system are developed following these amendments into the liver which leads to an inevitable portal hypertension $(9,13)$.

The central nervous system is damaged by toxic substances and bacteria which bypass the liver and damage appears as hepatic encephalopathy. These failures are manifested with epileptic seizures, arexia, polydipsia, diarrhea, intermittent vomiting, and most frequently with urolithiasis (ammonium urate). After nutrition hyperammonemia and increased serum bile acid are established by biochemistry examination of the blood and cristaluria of the urinalysis. In the neurologic review the following can be detected: hyperreactive episodes, circular movement, disorientations, temporary blindness, weakness, hypersalivation, hypersensitivity, followed by depression and coma $(1,6,9,13,14)$.

Varicose veins or varices on the surfaces of the different parenchymal organs are found by necropsy - on the liver, kidneys and other internal organs. This cavernous transformation in which venous vessels becomes enlarged, tortuous, twisted and extended with thin walls is developed by the permanent increase in portal pressure. These changes are associated with ascites. Lobular atrophy into portal regions, doubled in number arterioles and smaller ones, atresia or absent portal veins are all established by pathohistologic examination $(9,13,14)$. Esophageal varices, cirrhosis of the liver, choalangiohepatitis, neoplasias of the liver and arterio-venous fistulas are observed in human medicine in cases of extrahepatal PSS $(13,14)$.

\section{Case Description}

The dogs had shown similar clinical symptoms - epileptic seizures, arexia, polydipsia, diarrhea and vomiting. The exit for the animals is fatal, irrespective of the treatment, which was performed by us.

Case number 1: a female dog, 4 months, breed - Cocker Spaniel, with weight of $10 \mathrm{~kg}$. The hypoplastic portal vein (fig. 6), multiple veins which drained in jejunal loops and a colon descending directly into the caudal vena cava were observed through an autopsy (fig.1, 3 and 4). Some of these veins were infused into the left and right renal veins and also into the splenic vein (fig.1, 2 and 4). We also established communications of the intestinal veins with the left ovarian vein. Enlarged caudal vena cava with blood especially in the abdominal part was viewed by strong portal hypertension (fig. 2 and 3 ). The cavernous transformation or venous vessels on the diaphragmatic and visceral surfaces of the liver and the ventral surface of the kidneys were established (fig.2, 5 and 6). 


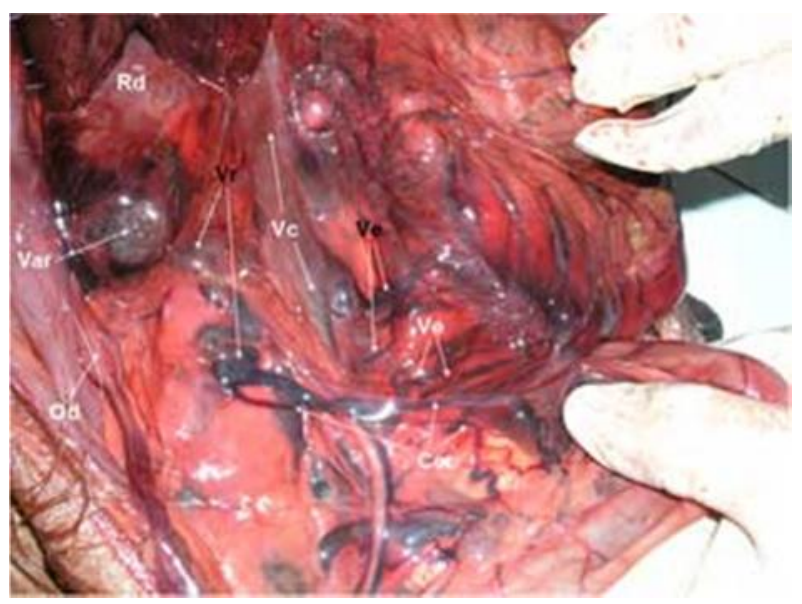

Fig. 1. Abdominal cavity of the dog - Rd - ren dexter, Od - ovarium dexter, Vc - vena cava caudalis, $\mathrm{Vr}$ - vena renalis dextra, Var - varices on the kidney's surface, Ve - veins draining intestines and connected directly with v. cava caudalis, Cer - veins draining intestines and connected with $\mathrm{c} \mathrm{v.}$ renalis dextra.

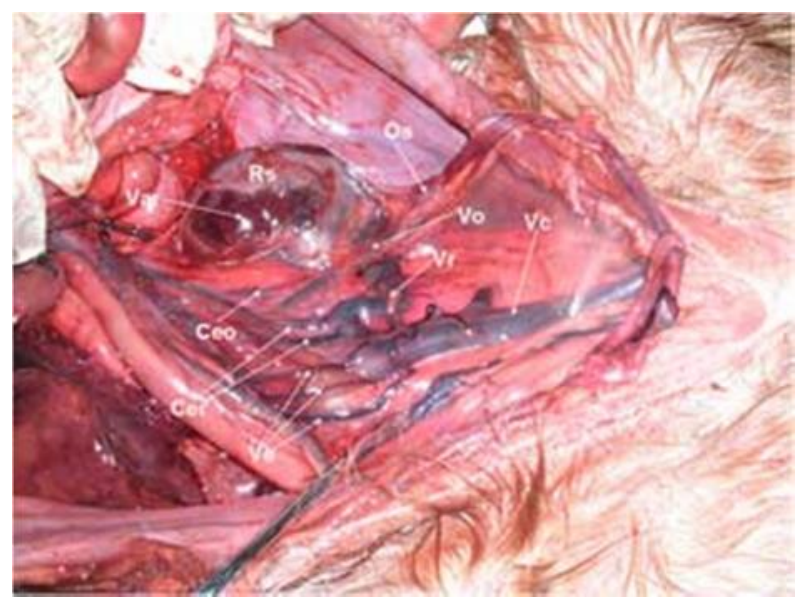

Fig. 2. Abdominal cavity of the dog - Rs - ren sinister, Os - ovarium sinister, Vc - vena cava caudalis is full with blood, $\mathrm{Vr}$ - vena renalis sinistra, Vo - vena ovarica sinistra, Var - varices on the kidney's surface, Ve - veins draining intestines and connected directly with v. cava caudalis, Cer veins draining intestines and connected with $\mathrm{c}$ v. renalis sinistra, Ceo - veins draining intestines and connected with $\mathrm{v}$. ovarica sinistra. 


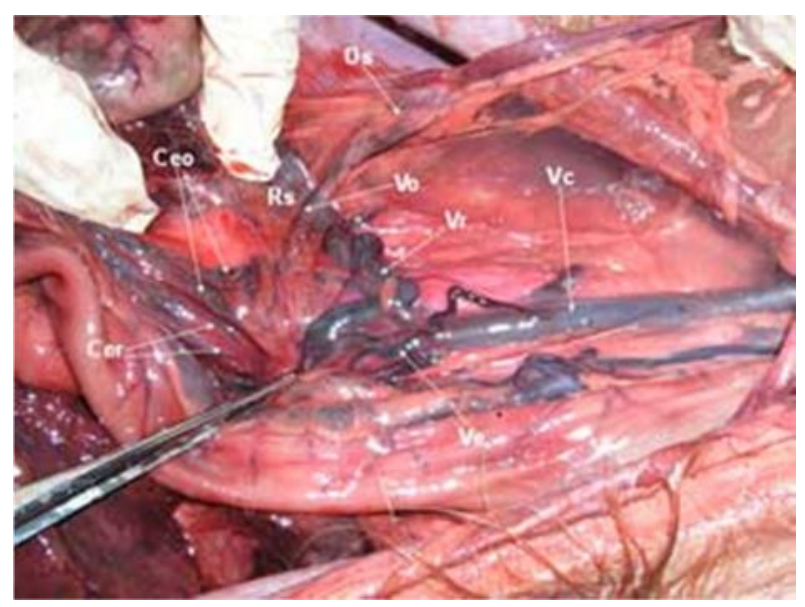

Fig. 3. Abdominal cavity of the dog - Rs - ren sinister, Os - ovarium sinister, Vc - vena cava caudalis is full with blood, $\mathrm{Vr}$ - vena renalis sinistra, Vo - vena ovarica sinistra, Ve - veins draining intestines and connected directly with v. cava caudalis, Cer - veins draining intestines and connected with $\mathrm{c}$ v. renalis sinistra, Ceo - veins draining intestines and connected with v. ovarica sinistra.

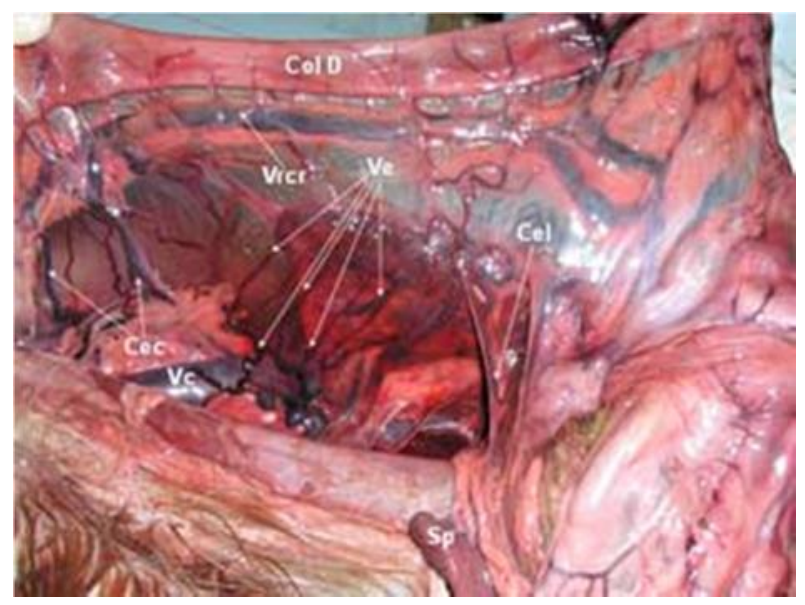

Fig. 4. Abdominal cavity of the dog - Col D - colon descendens, Sp - spleen, Vc - vena cava caudalis, Vrcr - vena rectalis cranialis, Ve - veins draining intestines and connected directly with v. cava caudalis, Cel - vena draining intestaines and connected with v. lienalis, Cec - veins draining colon descendens and connected directly with $v$ cava caudalis. 


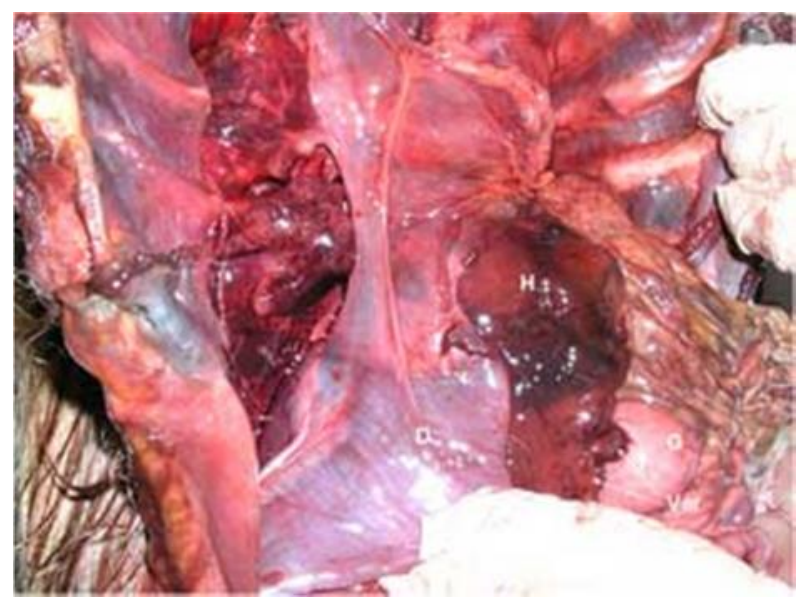

Fig. 5. Abdominal and thoracic cavity of the dog - - liver - diaphragm surface, G - gaster, D diaphragma, Var - varices on the diaphragm surface of the liver.

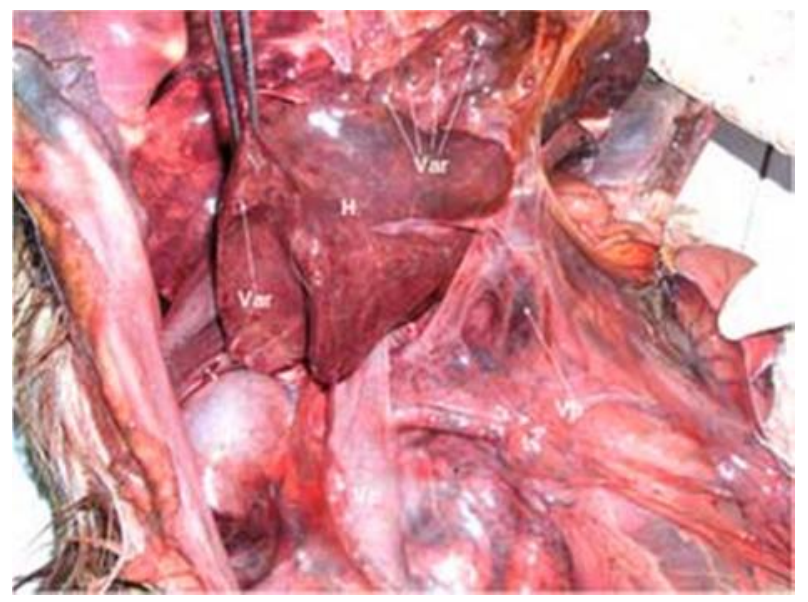

Fig. 6. Abdominal cavity of the dog - H - liver, Vc - vena cava caudalis, Vp - hypoplastic v. portae, Var - varices of the both surfaces of the liver.

Case number 2: a male dog, 6 months, breed - Rhodesian Ridgeback, with weight of $25 \mathrm{~kg}$. Normally, the ileocolic vein enters the cranial mesenteric vein after connecting to the middle of the colic vein. It is formed by the vessels from the transverse colon (fig. 9). This middle colic vein bypassed cranial mesenteric and portal veins without forming inlets into them (fig. 8). It entered through the porta of the liver and connected with branches of the hepatic veins which communicated directly with caudal vena cava (fig.7, 8 and 9). Near to the visceral surface of the liver before the entering of these veins which are enlarged, tortuous, twisted and extended - varicose veins were observed (fig. 7). 


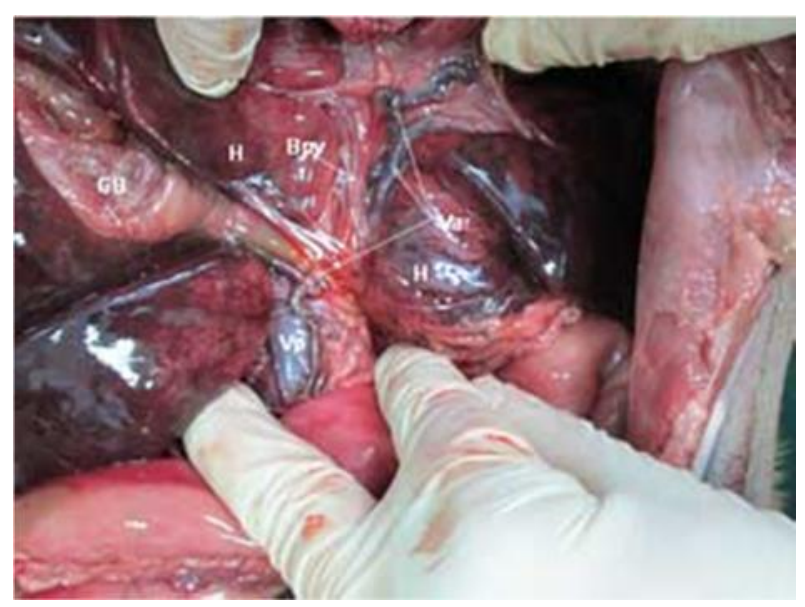

Fig. 7. Abdominal cavity of the dog - H - liver, GB - gallbladder, Vp - v, portae, Var - varicose veins of the visceral surfaces of the liver, Bpv - pathologic bypassing venous vessel which isn't connected with the portal vein.

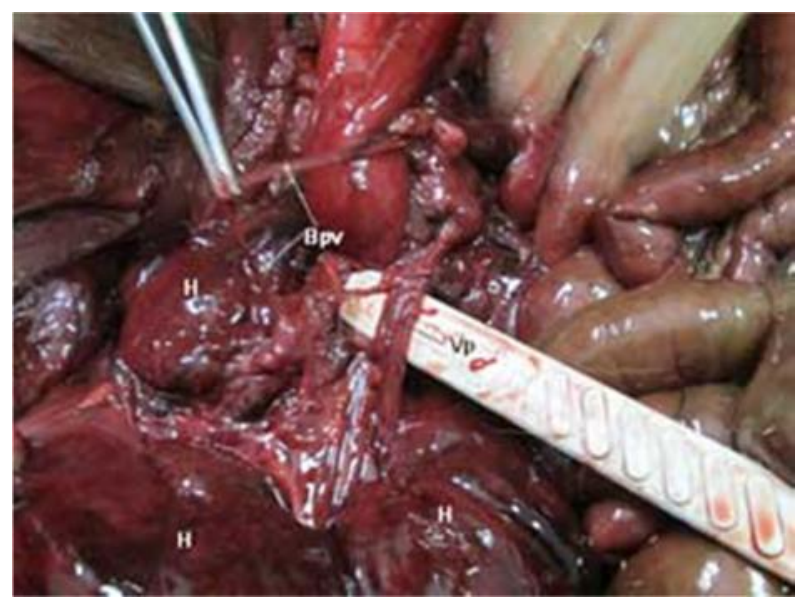

Fig. 8. Abdominal cavity of the dog - H - liver, Vp - v, portae, Bpv - pathologic bypassing venous vessel which isn't connected with the portal vein ( $v$. colica media). 


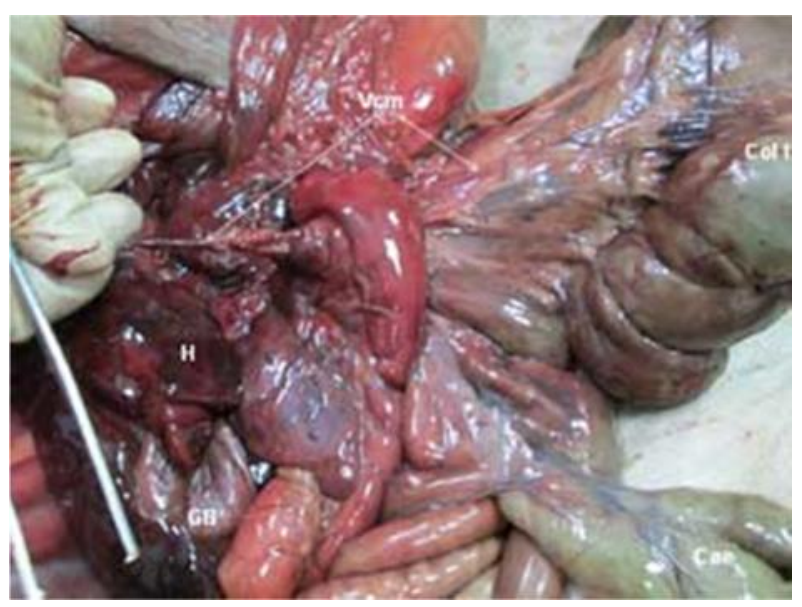

Fig. 9. Abdominal cavity of the dog - H - liver, GB - gallbladder, Col $\mathbf{t}$ - colon transversum, Cae caecum, Vcm - v. colica media.

\section{Discussion}

In large breed dogs intrahepatal PSS occurs which includes insufficient closing of the venous duct, which is normally present during fetal development and venous blood directly passes by the portal vessel system towards caudal vena cava which is reported by some authors $(1,6,9,13,14)$. In our study, the PSS in the large dog breed - Rhodesian Ridgeback was extrahepatal. In small breed dogs and cats single extrahepatal PSSs are found between the portal vein and caudal vena cava or the azygos vein and caudal vena cava by the same authors $(3,4,10,15)$. This claim was confirmed by us in the second of our cases where the middle colic vein did not flow into the cranial mesenteric vein (ileocolic vein) which is the largest inlet of the portal vein. However, Rhodesian Ridgeback is a large breed dog. This derivation vein was connected with branches of the hepatic veins into the liver parenchyma $(12,13)$.

The extrahepatal PSS in the middle dog breed - Cocker Spaniel was added by us. PSS in this case was multiple.

Communications of inlets of the portal veins - left gastric vein and the splenic vein - with caudal vena cava or the phrenicoabdominal vein in the dogs are reported (14). In the first case intestinal veins shunts with the renal and splenic veins in the dog were detected. These shunts are found in humans. Others bypasses are indicated in the human system - between intestinal veins and umbilical, paraumbilical, internal thoracic, caudal phrenical, pericardiacophrenical veins (8). The paraumbilical veins are connected with superior or inferior epigastric vein which are manifested with cavernous transformation of veins at body walls of the human - caput medusae $(2,8)$.

In the first case we observed a shunt of intestinal veins with the left ovarian vein in the dog. Communications between gonad veins connected with the inferior vena cava similar to as the mesoganadal shunt in humans are established by different authors (7).

The first clinical case was classified as a congenital PSS as there was hypoplasia of the portal vein, with developed of multiple collateral communications which conformed to what is described in some scientific papers $(1,6,7,12)$. 
The cavernous transformation on both the surfaces of the liver and of the kidneys were confirmed by us and were indicated as frequent pathologic findings $(8,9,12)$.

\section{Conclusion}

Extrahepatal PSS in the middle breed dog - Engle Cocker Spaniel was demonstrated which is classified as a congenital PSS with hypoplasia of the portal vein, which developed multiple collateral vessels as well as communications with veins of the portal vessels system with splenic, ovarian and renal veins. In this case of PSS it is impossible to operate. Amesogonadal shunt in the dog was observed. In the large breed dog - Rhodesian Ridgeback, we again observed an extrahepatal PSS . In this case, the shunt was single at the place where the middle colic vein bypasses the portal system and it was infused into the inlets of caudal vena cava.

For the proper diagnosing of PSS we recommend portography with a catheter input into the cranial mesenteric artery and rhythmic injection of the contrast medium.

\section{References}

1. Andrew E. Kyles. Portosystemic Shunts. Ettinger \& Feldman Textbook of Veterinary Internal Medicine. Client Information Sheet, 2005.

2. April E. Clinical anatomy. 3d ed.Williams and Wilkins. Maryland, USA, 1997.

3. Audell L, Jönsson LL, Lannek B. Congenital portacaval shunts in the dog. A description of three cases. Zentralbl Veterinaermed [A]. 1974; 21: 797-805.

4. Barrett RE, de Lahunta A, Roenick WJ, et al. Four cases of congenital portacaval shunt in the dog. J Small Anim Pract. 1976; 17: 71-85.

5. Evans HE, A. De Lahunta. Miller's Anatomy of the Dog. Fourth edition. Saunders\&Elseiver, St. Luis, Missouri, 2013.

6. Gallego C, Miralles M, Marín M, et al. Congenital Hepatic Shunts. Radio Graphics. 2004; 24: 755772.

7. Heung BK, James JP, Craig WL, et al. Mesogonadal Shunts with Extrahepatic Portal Vein Thrombosis and Variceal Hemorrhage. Liver Transplantation. 2005; 1; 1: 1389-1394.

8. Kenji I, Toshitaka T, Koichi M, Yoshihiro I. Transhepatic Portosystemic Shunts CT Appearance and Anatomic Correlation. American J of Roentgenology. 2000; 175:1.

9. McGavin, MD, Carlton W W,Zachary J.F. Thomson's special veterinary pathology. 3d ed. Mosby in St. Luis, 2001.

10. Payne JT, Martin RA, Constantinescu GM: The anatomy and embryology of portosystemic shunts in dogs and cats, Semin Vet Med Surg (Small Anim). 1900H ; 5:76-82. 
11. Schaller O. Illuistrated Veterinary Anatomical Nomenclature. Ferdinand Enke Verlag. Stuttgart, 2007.

12. Senzolo M, J. Tibbals, E. Cholongitas, C.K, et al... Transjugular intrahepatic portosystemic shunt for portal vein thrombosis with and without cavernous transformation. Aliment Pharm ter, 2006; 23; $6: 767-775$.

13. Soon MS, Chen YY, Yen HH. Spontaneous Intrahepatic Portosystemic Venous Shunt. J Gastroenterol Hepatol. Blackwell, 2006.

14. Susan E. J. Portosystemic shunts in dog and cat. Max's house. American College Veterinary Internal Medicine, USA.

15. Vitums A. Portosystemic communications in the dog. Acta Anat. 1959b; 39: 2271-2299.

\section{Corresponding author}

Georgi Georgiev

Sofia Bulgaria №1, Tsarigradsko shosse Blvd. 73 ,

tel: +359898743055

email: dr_gigeorgiev@abv.bg 